\title{
An Interpolation Series for Integral Functions
}

\author{
By Sheila Scott Macintyre
}

(Received 7th October, 1949. Read 4th November, 1949.)

1. The Gontcharoff interpolation series ${ }^{1}$

where

$$
\sum_{n=0}^{\infty} F^{(n)}\left(a_{n}\right) G_{n}(z)
$$

$$
G_{0}(z)=1, G_{n}(z)=\int_{a_{0}}^{z} d z^{\prime} \int_{a_{1}}^{z^{\prime}} d z^{\prime \prime} \ldots \int_{a_{n-1}}^{z^{(n-1)}} d z^{(n)} \quad(n>0),
$$

has been studied in various special cases. For example, if $a_{n}=a_{0}$ (all $n$ ), (1.0) reduces to the Taylor expansion of $F(z)$. If $a_{n}=(-1)^{n}$, J. M. Whittaker ${ }^{2}$ showed that the series (1.0) converges to $F(z)$ provided $F(z)$ is an integral function whose maximum modulus satisfies

$$
\varlimsup_{r \rightarrow \infty} \frac{\log M(r)}{r}<\frac{1}{4} \pi
$$

the constant $\frac{1}{4} \pi$ being the "best possible". In the case $\left|a_{n}\right| \leqslant 1$, I have shown ${ }^{3}$ that the series converges to $F(z)$ provided $F(z)$ is an integral function whose maximum modulus satisfies

$$
\varlimsup_{r \rightarrow \infty} \frac{\log M(r)}{r}<\cdot 7259,
$$

and 4 that while $\cdot 7259$ is not the "best possible" constant here, it cannot be replaced by a number as great as $\cdot 7378$.

In this paper, I consider a generalisation of Whittaker's result, namely the case in which $a_{n}=\omega^{n}$ where $|\omega|=1(\arg \omega \neq 0)$, and prove

THEOREM I. The series $\sum_{n=0}^{\infty} F^{(n)}\left(\omega^{n}\right) P_{n}(z)$, where $|\omega|=1, \arg \omega \neq 0$,

$$
P_{0}(z)=1, \quad P_{n}(z)=\int_{1}^{z} d z^{\prime} \int_{\omega}^{z^{\prime}} d z^{\prime \prime} \int_{\omega^{2}}^{z^{\prime \prime}} d z^{\prime \prime \prime} \ldots \int_{\omega^{n-1}}^{z^{(n-1)}} d z^{(n)} \quad(n>0),
$$

1 The notation used here differs from that adopted in 6 (Chapter III) in the omission of a factor $n$ ! from $G_{n}(z)$.

2 J. M. Whittaker, 5, 458.

3 S. S. Macintyre, 4.

S. S. Macintyre, 3. 
converges uniformly to $F(z)$ in any bounded region, provided $F(z)$ is an integral function whose maximum modulus satisfies

$$
\varlimsup_{r \rightarrow \infty} \frac{\log M(r)}{r}<\rho_{1},
$$

$\rho_{1}$ being the modulus of the smallest zero of the integral function $f(z, \omega)$ defined by the power series

$$
f(z, \omega)=\sum_{n=0}^{\infty} \omega^{\ln (n-1)} z^{n} / n !
$$

The constant $\rho_{1}$ is shown to be the "best possible" in this case, and it is evident that Whittaker's result follows as a special case, since

$$
f(z,-1)=\sin z+\cos z \text {. }
$$

It is possible to sharpen condition (1 . 1) of Theorem $\mathrm{I}$, and we prove

Theorem II. If we define $\omega, P_{n}(z), \rho_{1}$ as in Theorem $I$, the series $\sum_{n=0}^{\infty} F^{(n)}\left(\omega^{n}\right) P_{n}(z)$ converges uniformly to $F(z)$ in any bounded region, provided $F^{\prime}(z)$ is an integral function satisfying

$$
F(z)=O\left\{e^{\rho_{1}|z|} \phi\left(\rho_{1}|z|\right)\right\}
$$

where $\phi(z)$ is a function of $z$ such that $\sum_{k=1}^{\infty} \sqrt{k} \phi(k)$ is absolutely convergent.

2. Let the moduli of the zeros of $f(z, \omega)$ be arranged in a sequence $\rho_{n}$ in ascending order of magnitude. Differentiating (1.2) we have

$$
\begin{aligned}
\frac{\partial f}{\partial z} & =\sum_{1}^{\infty} \omega^{\frac{1 n(n-1)}{n}} z^{n-1} /(n-1) ! \\
& =\sum_{0}^{\infty} \omega^{\frac{1}{1 n(n+1)}} z^{n} / n ! \\
& =f(\omega z, \omega) .
\end{aligned}
$$

Consider

$$
\begin{aligned}
g(x, z) & =f\left(x z, \frac{I}{\omega}\right) / f\left(z, \frac{1}{\omega}\right) \\
& =\sum_{0}^{\infty} z^{n} Q_{n}(x) \quad\left(|z|<\rho_{1}\right) .
\end{aligned}
$$

Since

$$
g(x, 0) \equiv=1, \quad g(1, z) \equiv 1 \text {, }
$$

it follows that

$$
Q_{0}(x)=1 \quad \text { and } \quad Q_{n}(1)=0(n>0)
$$


Now it follows from (2.4), (2.5) that

$$
\frac{\partial g}{\partial x}=\sum_{1}^{\infty} z^{n} Q_{n}^{\prime}(x) \quad\left(|z|<\rho_{1}\right)
$$

and from (2.2), (2.3) that

$$
\begin{aligned}
\frac{\partial g}{\partial x} & =z g(x / \omega, z) \\
& =\sum_{0}^{\infty} z^{n+1} Q_{n}(x / \omega) .
\end{aligned}
$$

Hence, using (2.6), (2.7), we have

$$
Q_{n}{ }^{\prime}(x)=Q_{n-1}(x / \omega) \quad(n \geqslant 1) .
$$

It follows from $(2.5),(2.8)$ that

$$
Q_{n}(x)=\int_{1}^{x} d x^{\prime} \int_{1}^{x^{\prime} / \omega} d x^{\prime \prime} \int_{1}^{x^{\prime \prime} / \omega} d x^{\prime \prime \prime} \ldots \int_{1}^{x^{(n-1) / \omega}} d x^{(n)}
$$

or, by the transformations $\zeta^{(k)}=\omega^{k-1} x^{(k)}$,

Now, integrating

$$
\omega^{\frac{\hbar}{2 n(n-1)}} Q_{n}(x)=\int_{1}^{x} d \zeta^{\prime} \int_{\omega}^{\zeta^{\prime}} d \zeta^{\prime \prime} \cdots \int_{\omega n-1}^{\zeta(n-1)} d \zeta^{(n)}=P_{n}(x)
$$

$$
R_{n}(z)=\int_{1}^{z} d z^{\prime} \int_{\omega}^{z^{\prime}} d z^{\prime \prime} \ldots \int_{\omega^{n-1}}^{z^{(n-1)}} F^{(n)}\left(z^{(n)}\right) d z^{(n)}
$$

repeatedly by parts ${ }^{1}$, we find

$$
R_{n}(z)=F^{\prime}(z)-\sum_{r=0}^{n-1} F^{(r)}\left(\omega^{r}\right) P_{r}(z)
$$

Hence

$$
F(z)=\sum_{r=0}^{n-1} F^{(r)}\left(\omega^{r}\right) P_{r}(z)+R_{n}(z) \text {. }
$$

Let $C, \Gamma$ be the circles $|z|=\frac{1}{2} \rho_{1},|z|=\frac{1}{2}\left(\rho_{1}+\rho_{2}\right)$ respectively. From (2.4) we have

$$
Q_{n}(x)=\frac{1}{2 \pi i} \int_{C} \frac{g(x, z)}{z^{n+1}} d z .
$$

If $f\left(z, \frac{1}{\omega}\right)$ has $p$ zeros (denoted by $z_{1}, z_{2}, \ldots, z_{p}$ ) on $|z|=\rho_{1}$, then $g(x, z)$ has $p$ poles (at most) between $C$ and $\Gamma$, residues $A_{1}(x), A_{2}(x), \ldots, A_{p}(x)$ respectively, these residues being bounded for $x$ in any bounded region.

Now $|f(z, \omega)|$ has no zeros on $\Gamma$ and thus has a positive minimum on $\Gamma$ which will be denoted by $m$. Since $|f(z, \omega)|=\left|f\left(z, \frac{1}{\omega}\right)\right|$, we have, using

1 See J. M. Whittaker, 6, 39, for a detailed argument of this nature. 
(1.2) and (2.3),

$$
\begin{aligned}
\left|\frac{1}{2 \pi i} \int_{\Gamma} \frac{g(x, z)}{z^{n+1}} d z\right| & \leqslant e^{\frac{z}{\left(\rho_{1}+\rho_{2}\right)|x|} / m\left(\frac{\rho_{1}+\rho_{2}}{2}\right)^{n}} \\
& \leqslant B(x) /\left(\frac{\rho_{1}+\rho_{2}}{2}\right)^{n},
\end{aligned}
$$

where $B(x)$ is bounded for $x$ in any bounded region. Moreover the residue of $g(x, z) / z^{n+1}$ at $z=z_{8}(s=1,2, \ldots, p)$ is $A_{s}(x) / z_{s}^{n+1}$ and this is of absolute magnitude $\left|A_{s}(x)\right| \rho_{1}^{n+1}$. Now

$$
\frac{1}{2 \pi i} \int_{\Gamma} \frac{g(x, z)}{z^{n+1}} d z-\frac{1}{2 \pi i} \int_{C} \frac{g(x, z)}{z^{n+1}} d z
$$

is equal to the sum of the residues of $g(x, z) / z^{n+1}$ at the points $z_{1}, z_{2}, \ldots, z_{p}$ and it follows from $(2.12),(2.14)$ that

$$
\begin{aligned}
\left|Q_{n}(x)\right| & \leqslant \sum_{s=1}^{p} \frac{\left|A_{s}(x)\right|}{\rho_{1}^{n+1}}+B(x) /\left(\frac{\rho_{1}+\rho_{2}}{2}\right)^{n} \\
& \leqslant \frac{A(x)}{\rho_{1}^{n+1}}+B(x) /\left(\frac{\rho_{1}+\rho_{2}}{2}\right)^{n},
\end{aligned}
$$

where $A(x)$ and $B(x)$ are bounded for $x$ in any bounded region. On integrating both sides of the equation from 1 to $x / \omega$ we can show by induction that, for any integer $L$,

and thus

$$
\frac{x^{L}}{L ! \omega^{\frac{1}{2} L(L+1)}}=\sum_{r=0}^{L} \frac{Q_{L-r}(x / \omega)}{r ! \omega^{\frac{1}{r}(r-1)}}
$$

$$
\begin{aligned}
& S_{n, k}\left(\frac{x}{\omega}\right)=\int_{1}^{x / \omega} d x^{\prime} \int_{1}^{x^{\prime} / \omega} d x^{\prime \prime} \ldots \int_{1}^{x^{(n-1) / \omega}} d x^{(n)} \int_{0}^{x^{(n) / \omega}} d x^{(n+1)} \ldots \int_{0}^{x^{(k-1) / \omega}} d x^{(k)} \\
& =\frac{1}{\omega^{\frac{1}{((k-n)(k-n+1)}}} \int_{1}^{x / \omega} d x^{\prime} \int_{1}^{x^{\prime} / \omega} d x^{\prime \prime} \ldots \int_{1}^{x^{(n-1)} / \omega} \frac{x^{k-n}}{(k-n) !} d x \\
& =\sum_{r=0}^{k-n} \frac{Q_{k-r}(x / \omega)}{r ! \omega^{\text {tr(r-1)}}}
\end{aligned}
$$

from (2.16), making use of (2.5) and (2.8). Also, from (2.15) and (2.17) it follows that

$$
\begin{aligned}
\left|S_{n, k}\left(\frac{x}{\omega}\right)\right| & \leqslant A(x) \sum_{r=0}^{k-n} \frac{1}{r ! \rho_{1}^{k-r+1}}+B(x) \sum_{r=0}^{k-n} \frac{1}{r !}\left(\frac{2}{\rho_{1}+\rho_{2}}\right)^{k-n} \\
& \leqslant A(x) e^{\rho_{1}} / \rho_{1}^{k+1}+B(x) e^{1\left(\rho_{1}+\rho_{2}\right.} /\left(\frac{\rho_{1}+\rho_{2}}{2}\right)^{k}
\end{aligned}
$$


Again, if we use the transformations (2.9), the formula

$$
\omega^{\ddagger k(k-1)} S_{n, k}(x)=\int_{1}^{x} d \zeta^{\prime} \int_{\omega}^{\zeta} d \zeta^{\prime \prime} \ldots \int_{\omega^{n-1}}^{\zeta^{(n-1)}} d \zeta^{(n)} \int_{0}^{\zeta^{(n)}} d \zeta^{(n+1)} \ldots \int_{0}^{\zeta^{k-1)}} d \zeta^{(k)}
$$

arises from the definition of $S_{n, k}(x / \omega)$ in $(2.17)$.

3. From (2 10), (2 . 19), on expanding $F^{(n)}(z)$ in its Taylor series, we get

$$
\begin{aligned}
R_{n}(z) & =\int_{1}^{z} d z^{\prime} \int_{\omega}^{z^{\prime}} d z^{\prime \prime} \ldots \int_{\omega^{n-1}}^{z^{(n-1)}} \sum_{k=n}^{\infty} F^{(k)}(0) \frac{z^{(k-n)}}{(k-n) !} d z \\
& =\sum_{k=n}^{\infty} F^{(k)}(0) \omega^{\frac{1}{1} k(k-1)} S_{n, k}(z),
\end{aligned}
$$

as follows from (2.17) and (2.19). If $F(z)$ is an integral function, we have

$$
F^{(k)}(0)=\frac{k !}{2 \pi i} \int_{|\zeta|=k / \rho_{1}} \frac{F(\zeta)}{\zeta^{k+1}} d \zeta
$$

and using Stirling's approximation for $k$ !, if $F(z)$ satisfies (1.3), we have

$$
F^{(k)}(0)=O\left\{\rho_{1}^{k} \sqrt{k} \phi(k)\right\}
$$

Hence, from (2.18) and $(3.1), R_{n}(z)$ is less in modulus than the sum of the remainders of two convergent series and thus tends to zero as $n$ tends to infinity. From (2.11) it then follows that the interpolation series

$$
\sum_{r=0}^{\infty} F^{(r)}\left(\omega^{r}\right) P_{r}(z)
$$

converges uniformly to $F(z)$ in any bounded region provided $F(z)$ is an integral function satisfying (1.3). This completes the proof of Theorem II and hence of Theorem $\mathbf{I}$.

Let $z_{1}$, where $\left|z_{1}\right|=\rho_{1}$, be the zero of smallest modulus of $f(z, 1 / \omega)$. That the constant $\rho_{1}$ of Theorems I and II is the "best possible" is seen by taking $F(z)=f\left(z z_{1}, 1 / \omega\right)$ for which the maximum modulus $M(r)$ clearly satisfies

$$
\varlimsup_{r \rightarrow \infty} \frac{\log M(r)}{r}=\left|z_{1}\right|=\rho_{1} .
$$

Then, by (2.2), $F^{(n)}\left(\omega^{n}\right)=z_{1}{ }^{n} f\left(z_{1}, 1 / \omega\right)$ for all $n$. Thus for this function all the terms of the series (3.3) are identically zero. It should be noted that $\bar{z}_{1}$ is the zero of smallest modulus of $f(z, \omega)$.

The numerical value of $\rho_{1}$ has been calculated ${ }^{1}$ for $\arg \omega=\frac{2}{3} \pi, \frac{4}{5} \pi, \frac{3}{4} \pi$

1 See R. P. Boas, 1 and 2; S. S. Macintyre, 3, 
and the equivalent in radians of $136^{\circ}, 137^{\circ}$, the values of $\rho_{1}$ in these cases being approximately $\cdot 746, \cdot 7398, \cdot 7379, \cdot 7378$ and $\cdot 7378$ respectively.

\section{REFERENCES.}

1. R. P. Boas, Jr., "Functions of exponential type II," Duke Math. Journal, 11 (1944), 17-22.

2. —_, "Functions of exponential type IV," Duke Math. Journal, 11 (1944), 799.

3. Sheila Scott Macintyre, "An upper bound for the Whittaker constant $W$," Journal London Math. Soc., 32 (1947), 305-311.

4. - - " On the zeros of successive derivatives of integral functions," Trans. American Math. Soc., 62 (1949), 241-251.

5. J. M. Whittaker, "Lidstone's series and two-point expansions of analytic functions," Proc. London Math. Soc. (2), 36 (1932), 451-469.

6. - Interpolatory Function Theory (Cambridge, 1935).

\section{The Untversity,} Aberdeen. 